\title{
MODELING NONLINEAR ROAD TRAFFIC NETWORKS FOR JUNCTION CONTROL
}

\author{
TAMÁS PÉTER \\ Department of Control and Transport Automation \\ Budapest University of Technology and Economics (BME), Múegyetem rkp. 3, H-1111, Budapest, Hungary \\ e-mail: peter. tamas@mail.bme.hu
}

\begin{abstract}
The paper introduces a method of mathematical modeling of high scale road traffic networks, where a new special hypermatrix structure is intended to be used. The structure describes the inner-inner, inner-outer and outer-outer relations, and laws of a network area. The research examines the nonlinear equation system. The analysed model can be applied to the testing and planning of large-scale road traffic networks and the regulation of traffic systems. The elaborated model is in state space form, where the states are vehicle densities on a particular lane and the dynamics are described by a nonlinear state constrained positive system. This model can be used directly for simulation and analysis and as a starting point for investigating various control strategies. The stability of the traffic over the network can be analyzed by constructing a linear Lyapunov function and the associated theory. The model points out that in intersection control one must take the traffic density values of both the input and the output sections into account. Generally, the control of any domain has to take the density of input and output sections into consideration.
\end{abstract}

Keywords: mathematical modeling and control, traffic networks, nonlinear positive system, linear Lyapunov function.

\section{Introduction}

Arneson and Langbort (2009) discuss the control of positive distributed systems. These systems represent the flow-through dynamics of the incoming material in a network of interconnected reservoirs according to mass conservation laws and the underlying structure of the interconnection network (Bastin, 1999). Such models have been used to describe a variety of different systems including automobile or aircraft traffic flow, job-balancing in computer clusters (Fu et al., 2006) or any systems of connected reservoirs with natural constraints, such as irrigation networks (Cantoni et al., 2007). The paper by Arneson and Langbort (2009) focuses on linear techniques for the design of static routing parameters for single destination networks under the goal of minimizing the total delay time while satisfying additional delay or capacity constraints. Delay in an air traffic network is costly, with costs arising, e.g., from missed connections or extra fuel consumption associated with airborne delays. For this reason, many algorithms for air traffic flow management have the minimization of delay costs (at least in part) as an objective. Many articles discuss such systems (see, e.g., Bertsimas and Patterson, 1998; Krozel and Penny, 2006).
Arneson and Langbort (2009) present a Linear Programing (LP) based method to design routing parameters to minimize the total delay. They prove that this method minimizes delay over all choices of routing parameters ensuring that the closed loop system is stable, positive, conservative and exhibits user specified interconnection. They consider positive systems which can be described as a network of sections through which material can travel. The flow out of a given section can diverge and enter multiple subsequent sections, including itself, and similarly, flow from multiple sections can converge and enter one subsequent section. The authors presented three theorems which can be used to design static routing parameters for positive, conservative systems representing the flow of material through networks. Delay constraints and capacity constraints can also be enforced. Haddad et al. (2010) examined optimal steady-state control for isolated traffic intersections. A simplified isolated controlled vehicular traffic intersection with two movements is considered. A discrete-event max-plus model is proposed to formulate an optimization problem for the traffic lamp switching sequence. In the case when the criterion is a strictly monotone linear function of the queue lengths, the problem becomes a linear programming one. 
Webster (1958) investigated the undersaturated conditions for isolated traffic intersections, i.e., the total flow entering the intersection can pass through within the cycle duration. He derived an expression for the average delay per vehicle for a given movement, based on theoretical analysis and empirical results. Other papers (e.g., Mazloumi, 2008; Rouphail and Akcelik, 1992) aimed to improve the delay estimation formula for oversaturated conditions, while others proposed different models, methods, and strategies for controlling oversaturated isolated intersections (Chang and Lin, 2000; Gazis, 1964; Guardabassi et al., 1984; Improta and Cantarella, 1984; Kashani and Saridis, 1983; Michalopoulos and Stephanopoulos, 1978; Talmor and Mahalel, 2007) where the control objective was to minimize delays or to maximize the intersection capacity. With proper sensors it is easier to measure queue lengths than to estimate delays. Therefore, in the technical note by Haddad et al. (2010), the criteria are functions of the queue lengths inspired by Gazis and Potts (1963), so the time integral over the sum of all queue lengths at the intersection, or the so-called "total delay", is minimized. A necessary and sufficient condition for the steady-state control with constant cycle length was also derived by Haddad et al. (2010). The $N$-stage control problem was formulated. It was shown that the $N$-stage control problem can be solved by LP if the criterion $J$ is linear and strictly increasing. Furthermore, Nava-stage control can be used to bring the queue lengths to the optimum.

Modeling and control of urban and city traffic networks are important tools to avoid congestions and traffic jams (Peter and Bokor, 2010). Investigation of applications of various modeling and control paradigms aims at helping to operate the traffic network more efficiently by increasing the throughput of a network. In most cases, models are used for simulation of various road traffic scenarios and serve as analysis tools. Modeling for control usually needs lower complexity models than those used for analysis, and these are preferably described in state space forms. A well known class of control oriented models is based on the store-and-forward approach, which describes a queue building in front of a stop line (Drew, 1968; Gazis, 1976). This model is used in the TUC urban control strategy proposed by Diakaki et al. (2003), who designed and implemented a Linear Quadratic (LQR) control strategy. Another control approach was elaborated by Kulcsar et al. (2005) and Tettamanti et al. (2008), with the focus on the model predictive control concept. This approach allowed taking the green light constraints into consideration. Soft computing methods are also widely used in the analysis of traffic systems and transport problems (see Földesi and Botzheim, 2010; Harmati et al., 2007). Modern control methods offer powerful mathematical tools which can be successfully applied also in non-control oriented fields of vehicle dynamics, for ex- ample, in the crash process (Harmati et al., 2010).

\section{Mathematical model}

A common topic of research projects is macroscopic modelling, analysis and control of large-scale transport network processes. For this purpose, we propose a test methodology based on the theory of positive systems, where, in essence, the model is a macroscopic one. The first definition of positive systems was given by Luenberger (1979), stating that a positive system is the one in which the state variables are not negative. The majority of the analysed road traffic processes meet this requirement based on the original physical meaning of their states. Road traffic processes are usually described in the literature by setting up general linear systems of equations not exploiting the positive characteristics of the processes. We might think that the known properties of general linear systems are true in the case of positive systems as well, but they are not. The terms of the controllability and observability of positive systems are not derivable from the known methods applied in general systems.

The problem is particularly true when a non-negative co-domain is required not only for the states, but for the control input sign, too. Therefore, describing road system processes as pure positive systems is not a trivial task from the control engineering point of view. The control task in this case means that the system must be controlled from one state to another so that the states remain non-negative values during the transitions, too. The descriptions of the systems and controllability were given by Caccetta and Rumchev (2000), Farina and Rinaldi (2000), as well as Bacciotti (1983), Coxson and Shapiro (1987) and Valcher (1996).

According to Boothby (1982) and Sachkov (1997), regarding the real matrix $A$ applied in control theory the following theorem holds: The system is positive if and only if the matrix $A$ is a Metzler matrix, i.e., all of the elements not in the main diagonal are non-negative (the elements in the main diagonal may be arbitrary). In our

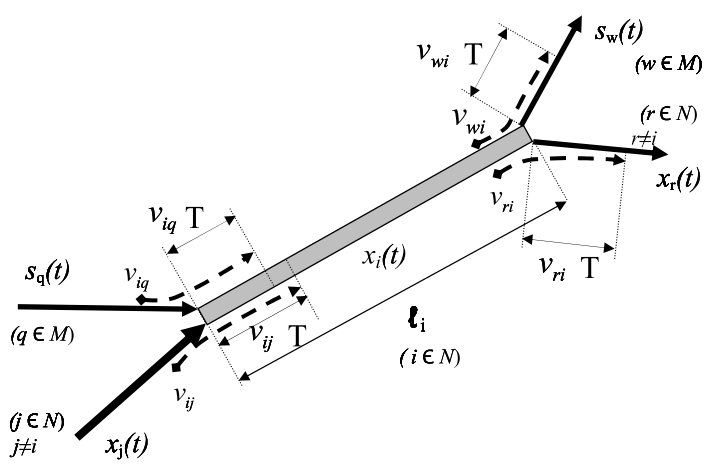

Fig. 1. Illustration of inner section modeling. 
case nonlinear positive systems are examined. The wellknown road traffic network models treat junctions or intersections as essential elements in models. This results in a graph which closely copies maps, while its apices are the junctions or intersections, and the arcs are the road sections connecting them. The optimal function of junctions is really important in the system. However, if we consider their role more thoroughly, they are the inevitable evil in the network. It would be ideal from the transportation point of view if less and less intersection traffic were there. Furthermore, if they did not exist, one could get from any point to another without intersection traffic. That obviously sounds absurd, but this thought leads us to another approach. It raises the question whether it is indeed necessary to have junctions as the focus of examinations.

The correct answer is that as far as transport is concerned, the focus of examination has to be placed on the whole of the network. The components of the network are, as an initial approach, the lanes and the parking spaces, and the parking lanes alongside the roads. The parking spaces and the parking lanes will be modeled as generalized sections of the network. These sections co-operate in the whole network and these components form the edges of the network graph. The edges of the controlled graph will represent dynamic relations among the co-operating sections. The relations between the sections are described by an interaction matrix. It takes all elements included in a particular map of the traffic network and all rules (including traffic lights) that determine how the traffic runs into consideration. The rules specify the traffic on the elements, and a set of conditions on how the traffic flows from one element to another. The map also contains important parameters, such as the length, width, the number of lanes, the number of vehicles that can be located in parking spaces, numeric figures of the allowed speed, which considered the parameters of the dynamical model. A junction does not appear as an individual component here, as the function of each junction is a part of the relation system. A particular component of the model (section) is illustrated in Fig.1.

Assuming that $h$ is the length of a unit-vehicle, the state of a section $i$ is defined as

$$
x_{i}(t)=\frac{n_{i}(t) \cdot h}{l_{i}}
$$

where $n_{i}$ denotes the number of vehicles on section $i, h$ and $l_{i}$ denote the average length of the vehicles and the length of this section, respectively. This definition results in a dimensionless quantity describing the ratio of the total length of vehicles moving on section $i$ and the length of this section. The state variables are positive and satisfy $0 \leq x_{i} \leq 1$.

The in-flow and out-flow vehicle traffic densities are denoted by $s_{k}, k \in M$, where the index set $M$ specifies the number of flows entering and leaving section $i$. In this model the (geometrical) vehicle density is positive, too, and satisfies $0 \leq s \leq 1$. It is a dimensionless quantity which measures the ratio of the total length of vehicles on a certain section and the length of that section.

To make a comparison with the conventional use of vehicle density definition $S$ with the dimension of the number of vehicles per km (Diakaki et al., 1998; Kachroo and Ozbay, 1999; Papageorgiu, 1991), this definition describes traffic density as the number of vehicles being on a given road section, in an amount $t$ of time. The dimensionless $s$ can be related to $S$ by using the length of the unit-vehicle $h$, measured in meters, as

$$
s=\frac{S \cdot h}{1000} .
$$

The parking spaces can be modeled as generalized sections of the network. Denote by $P_{i}$ a parking space where the number of vehicles that can be stored is denoted by $N_{i}$, and the number of vehicles parking there at a particular time instance $t$ is given by $n_{i}(t)$. Define the associated state variable as $x_{i}(t)=n_{i}(t) / N_{i}$ so that

$$
x_{i}(t)=\frac{n_{i}(t) \cdot h}{N_{i} \cdot h},
$$

and therefore a fictive section with length $l_{i}=N_{i} \cdot h$ can be defined and added to the network.

Our transport network model, consisting of $n$ pieces of inner sections, describes that route/urban transport system, which is located in a region bounded by a closed curve.

In this case, the $\left(H_{i}\right)$ vehicle densities evolving on the inner network are the system's features of condition, in the order of $x_{1}(t), x_{2}(t), x_{3}(t), \ldots, x_{n}(t)$. The model also uses the sub-network of the outer network $\left(H_{o}\right)$, which consists of such $m$ pieces of sections that have a direct relation with some inner sections. Vehicle densities evolving on them are indicated by $s_{1}(t), s_{2}(t), \ldots, s_{m}(t)$, which are known based on measurements. Our mathematical model depicting the network takes into consideration the inner relations of the network within the region and the outer relations of it outside the region in Fig. 2.

The traffic network under consideration will consist of $n$ inner sections and describes a route/urban transport system which is located in a region within a closed curve. The state vector $x(t)=\left[x_{1}(t), x_{2}(t), x_{3}(t), \ldots, x_{n}(t)\right]$ will include all the states of the inner elements and the variables $s$ will be considered measurable exogenous variables.

The state equation of the original physical model is the following nonlinear differential set of equations:

$$
\dot{x}=L^{-1}\left[K(x(t), s(t)) x(t)+K^{e x}(x(t), s(t)) s(t)\right],
$$

where $x \in \mathbb{R}^{n}, s \in \mathbb{R}^{m}, L=\operatorname{diag}\left\{l_{1}, \ldots, l_{n}\right\}$ contains the lengths of the inner sections $\left(\forall l_{i}>0, i=\right.$ 
$1,2, \ldots, n), K \in \mathbb{R}^{n \times n}, K^{e x} \in \mathbb{R}^{n \times m}$. In the model for any $x_{i}(i=1,2, \ldots, n)$ and $s_{k}(k=1,2, \ldots, m)$ stateparameters are dimensionless densities and take values in the interval $[0,1]$.

In the case of Eqns. (5), (8) and (15) the original physical model is written in the form of conventional state equations relative to control models.

On the basis of the following it is evident that $K$ is a Metzler matrix and $K^{e x}$ is a positive matrix, which does not contain negative elements.

The physical meaning of the elements $v_{i j}(i=$ $1,2, \ldots, n ; j=1,2, \ldots, n)$ of matrix $K$ is a velocity $(\mathrm{m} / \mathrm{s})$. In the case of $v_{i j}$ it means the transitional velocity from inner section $j$ to inner section $i(j \neq i)$, which is a non-negative value. On the main diagonal, $v_{j j}$ (which denotes the outflow velocities from inner section $j$ ) is the sum of outflow velocities to the inner and outer sections in the case of section $j$. These are non-positive values. To sum up, there are zeroes or negative values on the main diagonal of $K$, every other element is zero or positive; consequently, $K$ is a Metzler matrix.

The physical meaning of the elements $v_{i j}^{e x}(i=$ $1,2, \ldots, n ; j=1,2, \ldots, m)$ of matrix $K^{e x}$ is a velocity $(\mathrm{m} / \mathrm{s})$ as well. In the case of $v_{i j}^{e x}$ it means the transitional velocity from outer section $j$ to inner section $i$, which is a non-negative value. Consequently, in the case of $K^{e x}$, every element of the matrix is zero or positive.

In Eqns. (5) and (8), matrices $A, B_{s}$ in the discrete model and matrices $\tilde{A}, \tilde{B}_{s}$ in the continuous model are composed from matrices $K$ and $K^{e x}$ with linear transformation. In the following transformations the elements of matrices $K$ and $K^{e x}$ are multiplied by positive numbers and positive values are added to the elements on the main diagonal as well. The result of the transformation in the case of $A$ and $\tilde{A}$ is a Metzler matrix and in the case of $B_{s}$ and $\tilde{B}_{s}$ a positive matrix.

The state equations can be constructed by describing the traffic interactions among the sections including the effect of in- and out-flows $s$. The interactions will be effected by traffic lights as will be illustrated later. The

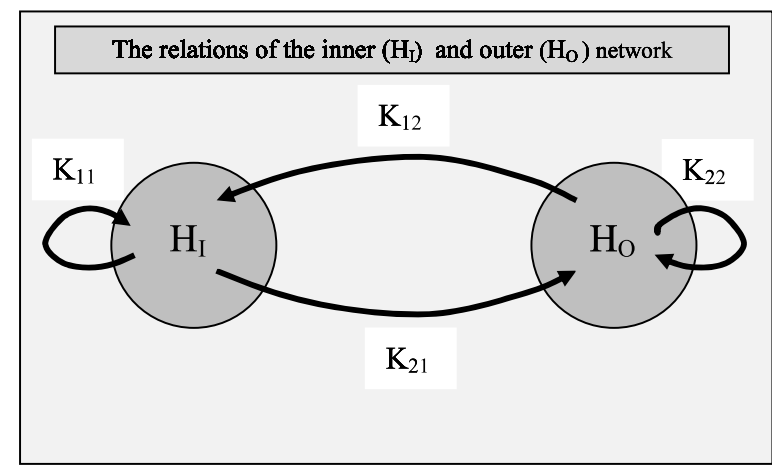

Fig. 2. Relations of the inner and the outer network. effects of green lights will be considered positive control inputs.

The state space model in discrete time can be expressed as

$$
x(t+1)=A(x(t), s(t)) x(t)+B_{s}(x(t), s(t)) s(t),
$$

where $0 \leq x(t) \leq 1$ and $0 \leq s(t) \leq 1, \forall t$, and

$$
\begin{aligned}
A(x(t), s(t)) & =L^{-1}[L+T K(x(t), s(t))], \\
B_{s}(x(t), s(t)) & \left.=L^{-1} T K^{e x}(x(t), s(t))\right],
\end{aligned}
$$

where $T$ is the model step.

Generally, $K_{i j}=k_{i j} v_{i j}(t), K_{i j}^{e x}=k_{i j}^{e x} v_{i j}^{e x}(t)$ and $v_{i j}(t)=f\left(x_{i}(t), x_{j}(t)\right)$ or $v_{i j}^{e x}(t)=f\left(x_{i}(t), s_{j}(t)\right)$. The system is a positive system, and the model is essentially a macroscopic model.

In continuous time one can construct the state equations in a similar form:

$$
\dot{x}(t)=\tilde{A}(x(t), s(t)) x(t)+\tilde{B}_{s}(x(t), s(t)) s(t),
$$

where

$$
\begin{aligned}
\tilde{A}(x(t), s(t)) & =L^{-1} K(x(t), s(t)), \\
\tilde{B}_{s}(x(t), s(t)) & =L^{-1} K^{e x}(x(t), s(t)) .
\end{aligned}
$$

Although our model is a special macroscopic model, with this method it can be used to determine the individual vehicle movements and the expected arrival times (Peter and Basset, 2009). The above mentioned problem requires using the calculus of variations. The solution is simplified by the fact that the number of the possible trajectories is finite. Using assumptions on the dependence of the vehicle speed on the traffic density, there are many options to specify the matrix function $\tilde{A}, \tilde{B}_{s}$; see, e.g., the works of Greenshields (1934) (linear) or Greenberg (1959) (logarithmic). In the foregoing investigation we use the Greenshields function that assumes a linear relation between speed and density in the form $v(x)=V_{\max }(1-x)$, where $V_{\text {max }}$ defines a maximum speed. Under this assumption the $(i, j)$-th elements of the matrices $\tilde{A}, \tilde{B}_{s}$ will depend on the speed $v(x)$ such that

$$
v_{i j}=\frac{l_{i} \cdot V_{\max }\left(1-x_{i}\right)+l_{j} \cdot V_{\max }\left(1-x_{j}\right)}{l_{i}+l_{j}} .
$$

Traffic constraints can be taken into consideration, too. If section $i$ is full, i.e., $x_{i}=1$, then no vehicles can pass from, e.g., section $j$ to $i$. This situation can be described by the function

$$
S\left(x_{i}\right)= \begin{cases}0 & \text { if } x_{i}=1 \\ 1 & \text { otherwise }\end{cases}
$$

Also, there are no vehicles passing from section $j$ if it is empty. This can be modeled by defining a function

$$
E\left(x_{j}\right)= \begin{cases}1 & \text { if } x_{j}>0, \\ 0 & \text { if } x_{j}=0 .\end{cases}
$$


The element of the matrices taking these situations and traffic light controls into consideration can now be written as

$$
\begin{aligned}
& v_{i j} \\
& =u_{i} S\left(x_{i}\right) \frac{l_{i} \cdot V_{\max }\left(1-x_{i}\right)+l_{j} \cdot V_{\max }\left(1-x_{j}\right)}{l_{i}+l_{j}} E\left(x_{j}\right)
\end{aligned}
$$

for all $t \geq 0$, where $u_{i}$ is the length of the green light associated with section $i,(i \neq j)$.

In the sections that have interactions with exogenous variables, the state variables $x_{i}$ will be replaced by $s_{i}$. With obvious rearrangements, the continuous time state equations can be written in the form

$$
\begin{aligned}
\dot{x}(t)= & \hat{A}(x(t), s(t)) x(t)+\hat{B}_{s}(x(t), s(t)) s(t) \\
& +\hat{B}_{u}(x(t), s(t)) u(t) .
\end{aligned}
$$

If there is no traffic light control in a given network, the last term is missing.

Let us examine the types of matrices $\hat{A}$ and $\hat{B}$ in Eqn. (15). This equation can be written with separation based on the original physical equation in the following form:

$$
\begin{aligned}
& \hat{A}=K(\underline{x}, \underline{s}, \underline{u}=\underline{0}), \\
& \hat{B}=K^{e x}(\underline{x}, \underline{s}, \underline{u}=\underline{0}) .
\end{aligned}
$$

$\hat{B}_{u} \in \mathbb{R}^{n \times q}$, where the $i$-th column $(i=1, \ldots, q)$ is

$$
\frac{\partial}{\partial u_{i}}(K(\underline{x}, \underline{s}, \underline{u}) \cdot \underline{x})+\frac{\partial}{\partial u_{i}}\left(K^{e x}(\underline{x}, \underline{s}, \underline{u}) \cdot \underline{s}\right) .
$$

In this case, the following is considered: In the original physical model $u_{i}(i=1,2, q)$ are non-negative control signals and multiplier factors of the matrix elements. Every element can be multiplied by only one $u_{i}$ factor. However, a factor $u_{i}$ can modify several elements.

In this procedure elements are omitted from the original $K$ Metzler matrix resulting in a matrix $\hat{A}$, which, consequently, is a Metzler matrix as well. In the same way elements are omitted from the positive matrix $K^{e x}$ resulting in a matrix $\hat{B}$, which hence is a positive matrix as well.

Since the matrices $\hat{A}, \hat{B}_{s}$ and $\hat{B}_{u}$ are functions of the measurable variable $s$ and the state variable $x$, this system is called a quasi Linear Parameter Varying (qLPV) system. In addition, it can be shown that in most situations (for fixed $x$, and $s$ ), the matrix $\hat{A}$ is a Metzler matrix and this qLPV system is positive. This leads to the investigation of equilibrium points, stability and control by using the theory of qLPV and of positive systems. There are few existing literature items that publish results on this class of systems.

\section{Equilibria and Lyapunov stability}

A nonlinear positive system can have many equilibrium points. Their stability can be investigated through Lyapunov theory using a linear Lyapunov function,

$$
V(x)=\sum_{i=1}^{n} f_{i} x_{i}
$$

For the discrete time system the condition $V(t+1)<$ $V(t)$ ensures asymptotic stability of the origin, (see, e.g., Luenberger, 1979). The examination of this expression generates a solution for the control law through the Lyapunov function, which gives an eligible solution for the asymptotic stability of the system and can dynamically be applied to the whole region as well as its critical subdomains.

In the following discussion we examine the stability of the origin of the state space using the continuous time model with no control input $u$. Assume that the constraints described by the functions $S, E$ are not active. For the autonomous system (8), the following result can be obtained.

Proposition 1. The system described by Eqn. (8) is stable at the origin if

$$
\sum_{j=1}^{n} \sum_{w=1}^{m} v_{w j}^{\text {out }} x_{j}>\sum_{j=1}^{m} \sum_{i=1}^{n} v_{i j}^{\text {inp }} s_{j}^{\text {inp }}, \quad \forall t>0,
$$

where

$$
v_{w j}^{\text {out }}=V_{\max } \frac{p_{w} \cdot\left(1-s_{w}^{\text {out }}\right)+l_{j} \cdot\left(1-x_{j}\right)}{p_{w}+l_{j}}
$$

and

$$
v_{i j}^{i n p}=V_{\max } \frac{l_{i} \cdot\left(1-x_{i}\right)+p_{j} \cdot\left(1-s_{j}^{i n p}\right)}{l_{i}+p_{j}},
$$

i.e., the aggregated out-flow traffic on the boundary is larger than the aggregated in-flow traffic.

Proof. The outline of the proof is the following. The derivative of the Lyapunov function is $\dot{V}(x)=$ $\sum_{i=1}^{n} f_{i} \dot{x}_{i}$. If we take $f_{i}=l_{i}$, i.e., the coefficients are the section lengths, then

$$
\dot{V}(x)=\sum_{i=1}^{n} l_{i} \dot{x}_{i}=l^{T} L^{-1}\left[K(x, s) x+K^{e x}(x, s) s\right] .
$$

Since $l^{T} L^{-1}=[1,1, \ldots, 1]$, the above function is the sum of the columns of the relation matrices $K(x, s)$, $K^{e x}(x, s)$. By construction, the elements on the main diagonal of $K(x, s)$ have the form

$$
v_{i i}=-\left[\left(\sum_{r=1, r \neq i}^{n} v_{r i}\left(x_{r}, x_{i}\right)+\sum_{w=1}^{m} v_{w i}\left(s_{w}, x_{i}\right)\right)\right] \text {, }
$$


where the first term is the negative of the sum of all the other elements in the column $i$. This shows that the sum of the elements in the $i$-th column of $K(x, s)$ is

$$
\sum_{w=1}^{m} v_{w j} x_{j}<0
$$

Similarly, the $i$-th column sum of the matrix $K^{e x}(x, s) s$ is obtained as $\sum_{i=1}^{n} v_{i j} s_{j}>0$. Then the time derivative of the Lyapunov function will be negative for all $t$ if the condition in (20) is satisfied.

Notice that it is simple to find a physical meaning of this Lyapunov function. Since the state is defined as $x_{i}(t)$ and $V(x)=\sum_{i=1}^{n} l_{i} x_{i}=h \sum_{i=1}^{n} n_{i}$, the value of this function is equal to the total length of the vehicles on the inner road network. The negative value of the derivative $\dot{V}$ ensures that the total number of vehicles, i.e., the total length of the road occupied by the vehicles, will decrease in the network. A trivial consequence is that the autonomous system $\left(S^{e x}=0\right)$ has always a stable equilibrium at the state space origin.

Therefore, the negative value of the derivative of $V(t)$, according to $t$, means a decrease in the total number of vehicles as well as a decrease in the total amount of the occupied road length in the inner road network.

This examination generates a solution for the control law through the Lyapunov function, which gives an eligible solution for the asymptotic stability of the system and can be dynamically applied to the whole region and to its critical sub-domains.

\section{Application example}

This section illustrates the construction of the state space model for a simple network (two-way road crossing with traffic lights) shown in Figs. 4-5. It consists of four inflow sections described by the exogenous variables $s_{1}, s_{3}$, $s_{5}, s_{7}$, eight states $x=\left[x_{1}, x_{2}, x_{3}, x_{4}, x_{5}, x_{6}, x_{7}, x_{8}\right]^{T}$,

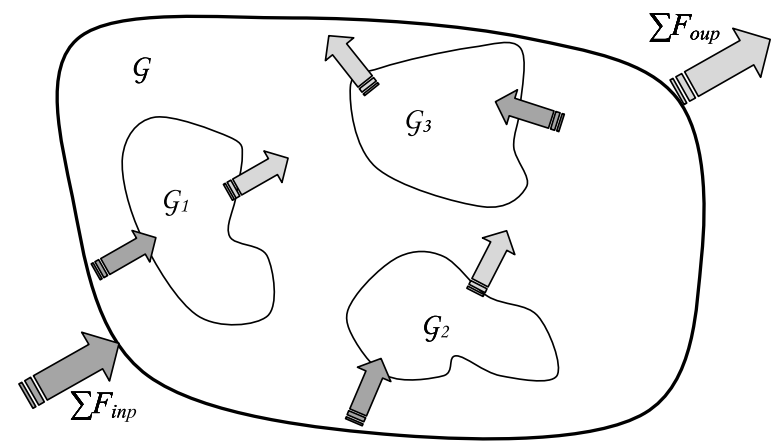

Fig. 3. Control law practising the Lyapunov function in the region and sub-regions. and four outflow sections; the associated variables are denoted by $s_{2}, s_{4}, s_{6}, s_{8}$. Assume that we have a single traffic light $u_{1}$ green at section 1 while red at section 2 , resulting in $u_{2}=1-u_{1}$.

In continuous time the state equation can be described as (15), where

- matrix $\hat{A}$ is a $8 \times 8$ sparse matrix whose nonzero elements are the following:

$$
\begin{aligned}
\hat{A}(2,2)= & -a o_{2,2}\left(s_{2}, x_{2}\right), \\
\hat{A}(2,7)= & a_{2,7}\left(x_{2}, x_{7}\right)-a_{2,7}\left(x_{2}, x_{7}\right) \alpha_{8,7}, \\
\hat{A}(3,3)= & -\alpha_{4,3} a_{4,3}\left(x_{4}, x_{3}\right)-a_{6,3}\left(x_{6}, x_{3}\right), \\
& +a_{6,3}\left(x_{6}, x_{3}\right) \alpha_{4,3}, \\
\hat{A}(4,3)= & \alpha_{4,3} a_{4,3}\left(x_{4}, x_{3}\right), \\
\hat{A}(4,4)= & -a o_{4,4}\left(s_{4}, x_{4}\right), \\
\hat{A}(6,3)= & a_{6,3}\left(x_{6}, x_{3}\right)-a_{6,3}\left(x_{6}, x_{3}\right) \alpha_{4,3}, \\
\hat{A}(6,6)= & -a o_{6,6}\left(s_{6}, x_{6}\right), \\
\hat{A}(7,7)= & -a_{2,7}\left(x_{2}, x_{7}\right)+a_{2,7}\left(x_{2}, x_{7}\right) \alpha_{8,7}, \\
& -\alpha_{8,7} a_{8,7}\left(x_{8}, x_{7}\right), \\
\hat{A}(8,7)= & \alpha_{8,7} a_{8,7}\left(x_{8}, x_{7}\right), \\
\hat{A}(8,8)= & -a o_{8,8}\left(s_{8}, x_{8}\right) ;
\end{aligned}
$$

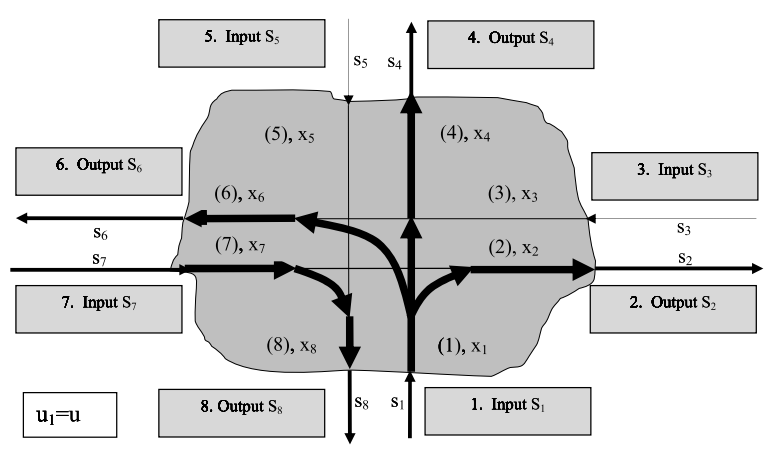

Fig. 4. Two-way road crossing with the traffic light $u_{1}=1$.

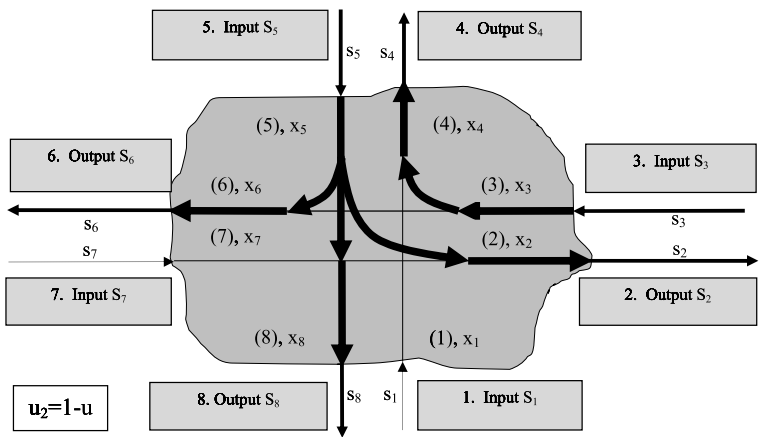

Fig. 5. Two-way road crossing with the traffic light $u_{1}=0$. 
- matrix $\hat{B}_{s}$ is an $8 \times 8$ sparse matrix whose nonzero elements are the following:

$$
\begin{aligned}
& \hat{B}_{s}(1,1)=a i_{1,1}\left(x_{1}, s_{1}\right), \\
& \hat{B}_{s}(3,3)=a i_{3,3}\left(x_{3}, s_{3}\right), \\
& \hat{B}_{s}(5,5)=a i_{5,5}\left(x_{5}, s_{5}\right), \\
& \hat{B}_{s}(7,7)=a i_{7,7}\left(x_{7}, s_{7}\right) ;
\end{aligned}
$$

- matrix $\hat{B}_{u}$ is equal to

$$
\left[\begin{array}{c}
\left(-\alpha_{2,1} a_{2,1}\left(x_{2}, x_{1}\right)-\left(1-\alpha_{2,1}\right) a_{4,1}\left(x_{4}, x_{1}\right)\right) x_{1} \\
\alpha_{2,1} a_{2,1}\left(x_{2}, x_{1}\right) x_{1}-\left(1-\alpha_{8,7}\right) a_{2,7}\left(x_{2}, x_{7}\right) x_{7} \\
\left(\alpha_{4,3} a_{4,3}\left(x_{4}, x_{3}\right)+\left(1-\alpha_{4,3}\right) a_{6,3}\left(x_{6}, x_{3}\right)\right) x_{3} \\
\left(1-\alpha_{2,1}\right) a_{4,1}\left(x_{4}, x_{1}\right) x_{1}-\alpha_{4,3} a_{4,3}\left(x_{4}, x_{3}\right) x_{3} \\
\left(-\alpha_{6,5} a_{6,5}\left(x_{6}, x_{5}\right)-\left(1-\alpha_{6,5}\right) a_{8,5}\left(x_{8}, x_{5}\right)\right) x_{5} \\
-\left(1-\alpha_{4,3}\right) a_{6,3}\left(x_{6}, x_{3}\right) x_{3}+\alpha_{6,5} a_{6,5}\left(x_{6}, x_{5}\right) x_{5} \\
\left(\left(1-\alpha_{8,7}\right) a_{2,7}\left(x_{2}, x_{7}\right)+\alpha 8,7 a_{8,7}\left(x_{8}, x_{7}\right)\right) x_{7} \\
\left(1-\alpha_{6,5}\right) a_{8,5}\left(x_{8}, x_{5}\right) x_{5}-\alpha_{8,7} a_{8,7}\left(x_{8}, x_{7}\right) x_{7}
\end{array}\right.
$$

where

$$
\begin{aligned}
a_{i, j}\left(x_{i}, x_{j}\right) & =S\left(x_{i}\right) v_{i, j}\left(x_{i}, x_{j}\right) E\left(x_{j}\right), \\
a o_{i, j}\left(s_{i}, x_{j}\right) & =S\left(s_{i}\right) v_{i, j}\left(s_{i}, x_{j}\right) E\left(x_{j}\right), \\
a i_{i, j}\left(x_{i}, s_{j}\right) & =S\left(x_{i}\right) v_{i, j}\left(x_{i}, s_{j}\right) E\left(s_{j}\right),
\end{aligned}
$$

and we adapt the following notation:

$$
\begin{aligned}
& \alpha_{i, j} \quad \text { the distribution rate }(j \rightarrow i), \\
& v_{i, j} \quad \text { the velocity of the flow }(j \rightarrow i), \text { defined by }(11), \\
& S \quad \text { defined by (12), } \\
& E \quad \text { defined by (13). }
\end{aligned}
$$

The performance variable $z$ is defined as the sum of the number of vehicles (see Fig. 6) that can be transmitted through the junction under all possible connections of road sections controlled (switched) by the traffic light (Peter and Bokor, 2010). In this example there are two

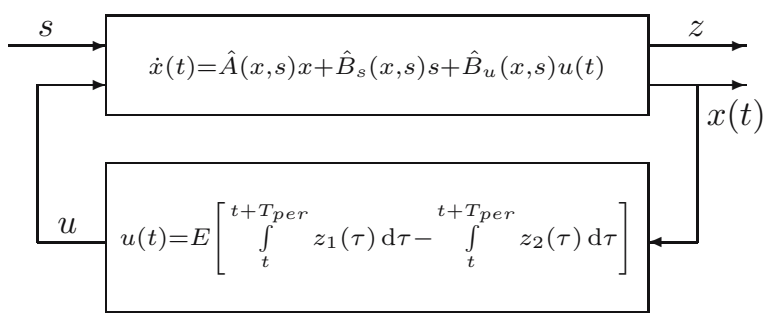

Fig. 6. Control loop of the traffic network control. possible switched connections, and the control objective is

$$
\int_{t_{0}}^{t} z(\tau) \mathrm{d} \tau \rightarrow \text { Max! }
$$

The control signal is a switching signal computed from the difference of the predicted number of transmitted vehicles in a given road section connection (section topology):

$$
\begin{aligned}
& z_{1}(t)=\sum_{i=1}^{4} N_{2 i-1}^{\text {Inp }}(t)+\sum_{i=1}^{4} N_{2 i}^{\text {Outp }}(t)+N_{1}(t), \\
& z_{2}(t)=\sum_{i=1}^{4} N_{2 i-1}^{\text {Inp }}(t)+\sum_{i=1}^{4} N_{2 i}^{\text {Outp }}(t)+N_{2}(t),
\end{aligned}
$$

where the number of transmitted vehicles in the period $\mathrm{d} \tau$ is

$$
\sum_{i=1}^{4} N_{2 i-1}^{\text {Inp }}(t), \quad \sum_{i=1}^{4} N_{2 i}^{\text {Outp }}(t), \quad N_{1}(t), \quad N_{2}(t),
$$

$$
\begin{aligned}
N_{1}(t)= & N_{12}(t)+N_{14}(t)+N_{56}(t)+N_{58}(t), \\
N_{2}(t)= & N_{34}(t)+N_{36}(t)+N_{72}(t)+N_{78}(t), \\
z(t)= & \sum_{i=1}^{4} N_{2 i-1}^{\text {Inp }}(t)+\sum_{i=1}^{4} N_{2 i}^{\text {Outp }}(t) \\
& +N_{1}(t) \cdot u(t)+N_{2}(t) \cdot(1-u(t)),
\end{aligned}
$$

and

$$
u(t)=E\left[\int_{t}^{t+T_{\text {per }}} z_{1}(\tau) \mathrm{d} \tau-\int_{t}^{t+T_{\text {per }}} z_{2}(\tau) \mathrm{d} \tau\right],
$$

where $E(x)=1$ if $x>0$ and $E(x)=0$ if $x \leq 0$ (see Fig. 6).

It can be deduced that this control is in a state feedback form and results in a switched (in this case bimodal) state dependent switching control system. The result of the simulations shows an increased number of transmitted vehicles as shown in Fig. 7, when compared with the traditional equally partitioned green light control strategy. When applying the switching control strategy, one can impose more conditions on $u$, like a minimum dwell time and a constraint on the periodicity.

In a generic case, considering an intersection of $n$ phases, the control $u_{k}(t)=1$ of the $k$-th phase is realized when the phase possesses the highest amount of $N_{k}$, the pre-calculated vehicle transmission. The control signal can be explicitly formulated mathematically if all phases possess different transfer rates:

$$
u_{k}(t)=\prod_{i=1 ; i \neq k}^{n} E\left(N_{k}-N_{i}\right), \quad k=1,2, \ldots, n .
$$

In this case, $u_{k}(t)=1$, and $u_{i}(t)=0 . \quad(i \neq k, i=$ $1,2, \ldots, n)$. 


\section{Summary}

The model can be applied to analyze and design largescale road traffic networks by simulations and to investigate control strategies in traffic systems. A special macroscopic model was used. Thus, mathematical models leading to partial differential equation systems can be avoided. Junction does not play a quintessential role in our special model, i.e., there are sections that either co-operate or not. The parallel lanes have an effect on each other. This interaction, which refers to depending on each other or disturbing each other, influences the density and speed of vehicles moving on parallel lanes. The incoming traffic also has an effect on the right and left lanes. This interaction manifests itself in the disturbance caused by passing each other. The defined parking spaces as well as the parking lanes alongside the roads are modeled as generalized sections in the network, and the vehicles parking there also interact with network sections. In the proposed model the vehicle density refers to the ratio of the total length of vehicles on a given section and the length of this section. Our road traffic model examines the vehicle density on the sections of a road network located within a closed curve. The vehicle flows into and out of the region are considered to be measurable. The in-flow vehicle densities measured on the boundaries of road sections act as exogenous signals. The densities $x_{i}(t)$ appearing on the road sections of the region are the system states.

The state equations derived from the operation of this road network are in the form of a positive qLPV system. Stability analysis of the origin of the state space was performed using Lyapunov theory. In addition, simulations under various traffic situations show that the model can also serve for designing traffic light control to increase the throughput of traffic networks.

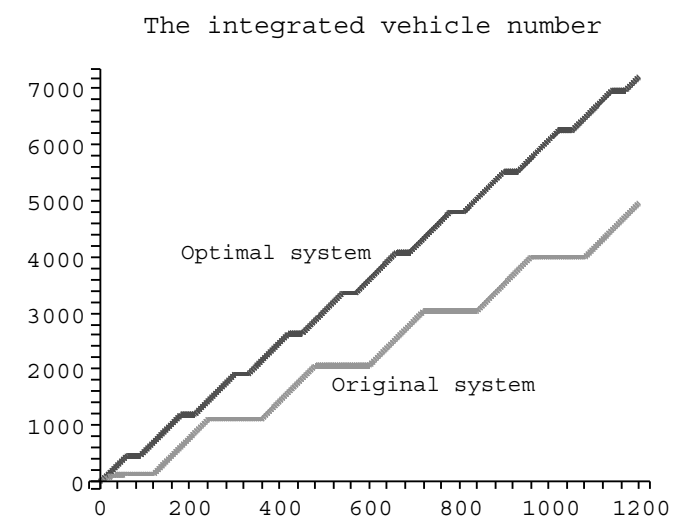

Fig. 7. Traffic flow through: the number of transmitted vehicles, two-way road crossing with traffic lights: the original and the system.

\section{Conclusions}

The research is expected to result in a new modelling methodology, by which the basic task is to create a more efficient model that describes and manages dynamic network systems of all sizes. In the course of this, elements must be determined of which a road transport system of any size can be built.

The other expected result of the research is that the new model leads to positive nonlinear systems theory (instead of the former traditional map-graph approach) in the field of mathematics. Solving large network problems, a new domain-level control principle and optimization using the Lyapunov function can be realized.

The research of large traffic networks is, besides the theoretical discussion, of major practical importance, too. There are significant economic interests worldwide in designing the optimum flow of vehicles and the optimal routes, as well as increasing safety. An expected practical result of the research is that the model will provide instructions for traffic control centres to avoid traffic jams based on real-time measurements (but by calculations faster than real time).

The model will also provide instructions for traffic control centres to avoid traffic congestions or environmental damages based on real-time vehicle density or emission measurements within a range. It will provide information for the vehicles to select an optimal and safe traffic route through a domain based on pre-calculations to avoid traffic jams.

Thanks to the available network IT equipments and many on-vehicle electronic and electromechanical components the majority of the targets are now achievable.

From the mathematical point of view the linear Lyapunov function applied in positive systems is certainly not a new result in itself. The new result in our case is the physical content of the Lyapunov function, which defines the length of all vehicles on a network bounded by an arbitrary closed curve. This result provides new possibilities in realizing the optimal control on the domain level.

\section{Acknowledgment}

The presented research work is sponsored by the OTKA CNK 78168 project, as well as the Control Engineering Research Group of the HAS at the Budapest University of Technology and Economics. This work is connected to the scientific program of the Development of quality-oriented and harmonized $R+D+I$ strategy and functional model at $B M E$ project. This project is supported by the New Hungary Development Plan (project ID: TMOP-4.2.1/B09/1/KMR-2010-0002). 


\section{References}

Arneson, H. and Langbort, C. (2009). Linear programming based routing design for a class of positive systems with integral and capacity constraints, Proceedings of the 1st IFAC Workshop on Estimation and Control of Networked Systems, Venice, Italy, pp. 352-357.

Bacciotti, A. (1983). On the positive orthant controllability of two-dimensional bilinear systems, System Control Letters 3(1): 53-55.

Bastin, G. (1999). Issues in Modeling and Control of Massbalanced Systems, in D. Aeyels, F. Lamnabhilagerrigve and A. van der Schaft (Eds.), Stability and Stabilization of Nonlinear Systems, Lecture Notes in Control and Information Sciences, Vol. 246, Springer, London, pp. 53-74.

Bertsimas, D. and Patterson, S. (1998). The air traffic flow management problem with en route capacities, Operations $R e$ search 46(3): 406-422.

Boothby, W.M. (1982). Some comments on positive orthant controllability of bilinear systems, SIAM Journal of Control and Optimization 20(5): 634-644.

Caccetta, L. and Rumchev, V. (2000). A survey of reachability and controllability for positive linear systems, Annals of Operations Research 98(1): 101-122.

Cantoni, M., Weyer, E., Li, Y., Ooi, S., Mareels, I. and Ryan, M. (2007). Control of large-scale irrigation networks, Proceedings of the IEEE 95(1): 75-91.

Chang, T. and Lin, J. (2000). Optimal signal timing for an oversaturated intersection, Transportation Research $B$ 34(6): 471-491.

Coxson, P. and Shapiro, H. (1987). Positive input reachability and controllability of positive systems, Linear Algebra and Its Applications 94: 35-53.

Diakaki, C., Dinopoulou, V., Aboudolas, K., Papageorgiou, M., Ben-Shabat, E., Seider, E., and Leibov, A. (2003). Extensions and new applications of the traffic control strategy TUC, TRB 2003 Annual Meeting, Washington, DC, USA.

Diakaki, C., Papageorgiou, M. and McLean, T. (1998). Integrated traffic-responsive urban control strategy, IN-TUC: Application and Evaluation in Glasgow. DACCORD Workshop on Advanced Motorway Traffic Control, Lancaster $U K$, pp. 46-55.

Drew, D.R. (1968). Traffic Flow Theory and Control, McGrawHill Book Company, New York, NY.

Farina, L. and Rinaldi, S. (2000). Positive Linear Systems: Theory and Applications, Wiley, New York, NY.

Földesi, P. and Botzheim, J. (2010). Modeling of loss aversion in solving fuzzy road transport traveling salesman problem using eugenic bacterial memetic algorithm, Memetic Computing 2(4): 259-271.

Fu, Y., Wang, H., Lu, C. and Chandra, R.S. (2006). Distributed utilization control for real-time clusters with load balancing, Proceedings of the 27th IEEE International Real-Time Systems Symposium, Rio de Janeiro, Brazil, pp. 137-146.

Gazis, D. (1964). Optimum control of a system of oversaturated intersections, Operations Research 12(6): 815-831.
Gazis, D.C. (1976). Optimal control of oversaturated store-andforward transportation networks, Transportation Science 10(1): 1-9.

Gazis, D. and Potts, R. (1963). The oversaturated intersection, Proceedings of the 2nd International Symposium on Theory of Traffic Flow, London, UK, pp. 221-237.

Greenberg, H. (1959). An analysis of traffic flow, Operations Research 7(1): 79-85.

Greenshields, B. (1934). A study of traffic capacity, Proceedings of the Highway Research Board 14: 448-477.

Guardabassi, G., Locatelli, A. and Papageorgiou, M. (1984). A note on the optimal control of an oversaturated intersection, Transportation Research B 18(2): 111-113.

Haddad, J., De Schutter, B., Mahalel, D., Ioslovich, I. and Gutman, P. (2010). Optimal steady-state control for isolated traffic intersections, IEEE Transactions on Automatic Control 55(11): 2612-2617.

Harmati, I., Orbán, G. and Várlaki, P. (2007). TakagiSugeno fuzzy control models for large scale logistics systems, Proceedings of the IEEE International Symposium on Computational Intelligence and Intelligent Informatics (ISCIII'07), Agadir, Morocco, pp. 199-203.

Harmati, I., Rövid, A. and Várlaki, P. (2010). Approximation of force and energy in vehicle crash using LPV type description, WSEAS Transactions on Systems 9(7): 734-743.

Improta, G. and Cantarella, G. (1984). Control systems design for an individual signalised junction, Transportation Research B 18(2): 147-167.

Kachroo, P. and Ozbay, K. (1999). Feedback Control Theory for Dynamic Traffic Assignment, Springer, London.

Kashani, H. and Saridis, G. (1983). Intelligent control for urban traffic systems, Automatica 19(2): 191-197.

Krozel, J. Jakobovits, R. and Penny, S. (2006). An algorithmic approach for airspace how programs, Air Traffic Control Quartelly 14(3): 203-229.

Kulcsar, B., Varga, I. and Bokor, J. (2005). Constrained split rate estimation by moving horizon, 16th IFAC World Congress, Prague, Czech Republic, Vol. 16, Part I, p. 2035.

Luenberger, D.G. (1979). Introduction to Dynamical Systems. Theory, Models and Applications, John Wiley and Sons, New York, NY.

Mazloumi, E. (2008). A new delay function for signalised intersections, Road Transportation Research 17(3): 3-12.

Michalopoulos, P. and Stephanopoulos, G. (1978). Optimal control of oversaturated intersections: Theoretical and practical considerations, Traffic Engineering Control 19(5): 216221.

Papageorgiu, M. (1991). Concise Encyclopedia of Traffic and Transportation Systems, Pergamon Press, Oxford.

Peter, T. and Basset, M. (2009). Application of new traffic models for determine optimal trajectories, International Forum on Strategic Technologies (IFOST 2009), Ho Chi Minh, Vietnam, pp. 89-94. 
Peter, T. and Bokor, J. (2010). Modeling road traffic networks for control, Annual International Conference on Network Technologies and Communications, NTC2010, Phuket Beach Resort, Thailand, pp. 18-22.

Rouphail, N. and Akcelik, R. (1992). Oversaturation delay estimates with consideration of peaking, Transportation Research Rec 1365: 71-81.

Sachkov, Y.L. (1997). On positive orthant controllability of bilinear systems in small codimensions, SIAM Journal of Control and Optimization 35(1): 29-35.

Talmor, I. and Mahalel, D. (2007). Signal design for an isolated intersection during congestion, Operation Research Soc 58(4): 454-466.

Tettamanti, T., Varga, I., Kulcsár, B and Bokor, J. (2008). Model predictive control in urban traffic network management, 16th Mediterranean Conference on Control and Automation, Ajaccio, France, pp. 1538-1543.
Valcher, M. (1996). Controllability and reachability criteria for discrete-time positive systems, International Journal of Control 65(3): 511-536.

Webster, F. (1958). Traffic signal settings, Technical report, Great Britain Road Research Lab., London.

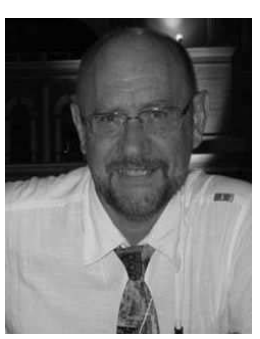

Tamás Péter, M.Sc. degree in mechanical engineering (Budapest University of Technology and Economics (BME), Hungary (1972)), doctorate in mathematics (1978), Ph.D. (1998). His research fields include non-linear stochastic dynamical systems, mathematical modeling, analysis and optimization, computer mathematics, equivalence classes of vehicle vibration systems, stochastic vehicle dynamics, road traffic and logistic models, and their applications, mathematical analysis and optimal control of large scale road traffic networks. Since 1972 he has been conducting research and teaching at BME.

Received: 8 April 2011

Revised: 9 October 2011

Re-revised: 21 December 2011 